Article

\title{
Optimization Technology of the LHS-1 Strain for Degrading Gallnut Water Extract and Appraisal of Benzene Ring Derivatives from Fermented Gallnut Water Extract Pyrolysis by Py-GC/MS
}

\author{
Chengzhang Wang ${ }^{1,2,3,4,5, *}$ and Wenjun $\mathrm{Li}^{1,2,3,4,5}$ \\ 1 Institute of Chemical Industry of Forest Products, Chinese Academy Forestry, Nanjing 210042, China; \\ liwenjun611@163.com \\ 2 National Engineering Laboratory for Biomass Chemical Utilization, Nanjing 210042, China \\ 3 Key and Open Laboratory on Forest Chemical Engineering, State Forestry Administration, \\ Nanjing 210042, China \\ 4 Key Laboratory of Biomass Energy and Material, Nanjing 210042, China \\ 5 Institute of New Technology of Forestry, Chinese Academy Forestry, Beijing 100091, China \\ * Correspondence: wangcz@icifp.cn; Tel./Fax: +86-025-8548-2471
}

Received: 31 October 2017; Accepted: 11 December 2017; Published: 20 December 2017

\begin{abstract}
Gallnut water extract (GWE) enriches $80 \sim 90 \%$ of gallnut tannic acid (TA). In order to study the biodegradation of GWE into gallic acid (GA), the LHS-1 strain, a variant of Aspergillus niger, was chosen to determine the optimal degradation parameters for maximum production of GA by the response surface method. Pyrolysis-gas chromatography-mass spectrometry (Py-GC/MS) was first applied to appraise benzene ring derivatives of fermented GWE (FGWE) pyrolysis by comparison with the pyrolytic products of a tannic acid standard sample (TAS) and GWE. The results showed that optimum conditions were at $31^{\circ} \mathrm{C}$ and $\mathrm{pH}$ of 5 , with a 50-h incubation period and $0.1 \mathrm{~g} \cdot \mathrm{L}^{-1}$ of TA as substrate. The maximum yields of GA and tannase were $63 \sim 65 \mathrm{mg} \cdot \mathrm{mL}^{-1}$ and $1.17 \mathrm{U} \cdot \mathrm{mL}^{-1}$, respectively. Over 20 kinds of compounds were identified as linear hydrocarbons and benzene ring derivatives based on GA and glucose. The key benzene ring derivatives were 3,4,5-trimethoxybenzoic acid methyl ester, 3-methoxy-1,2-benzenediol, and 4-hydroxy-3,5-dimethoxy-benzoic acid hydrazide.
\end{abstract}

Keywords: gallnut water extract; tannic acid; benzene ring derivatives; biodegradation; Py-GC/MS

\section{Introduction}

Gallnut is an abnormal gall in China produced by parasitic aphids on the host trees of Rhus chinensis, Rhus potaninii, or Rhus punjabensis, etc. Chinese gallnut can be divided into the three categories of Du-ensiform gall, horned gall, and gall flowers, according to aphid species and host differences. Du-ensiform gall enriches 55 65\% of tannin (Figure 1), and is the most traditional and important raw material for producing gallnut water extract (GWE) which contains 80 90\% tannin. GWE has been used to produce gallic acid through the hydrolysis of alkali, and acid catalysis. However, chemical degradation of GWE not only provides very low yields of gallic acid, but causes serious environmental pollution and equipment corrosion. Therefore, it is urgent to probe more environmentally friendly degradation technology in order to produce gallic acid and its derivatives $[1,2]$. 


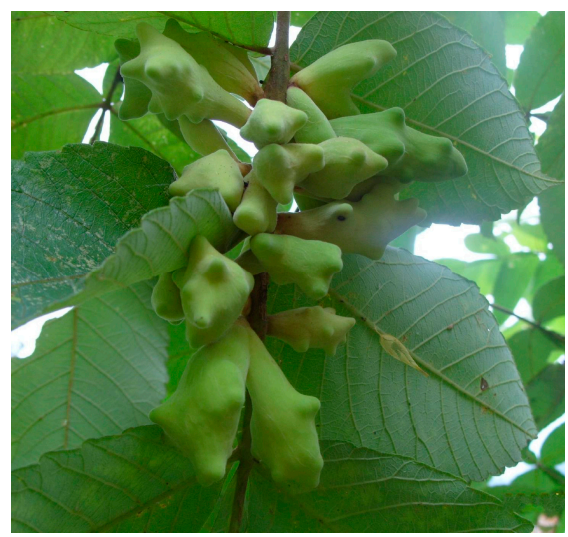

(a)

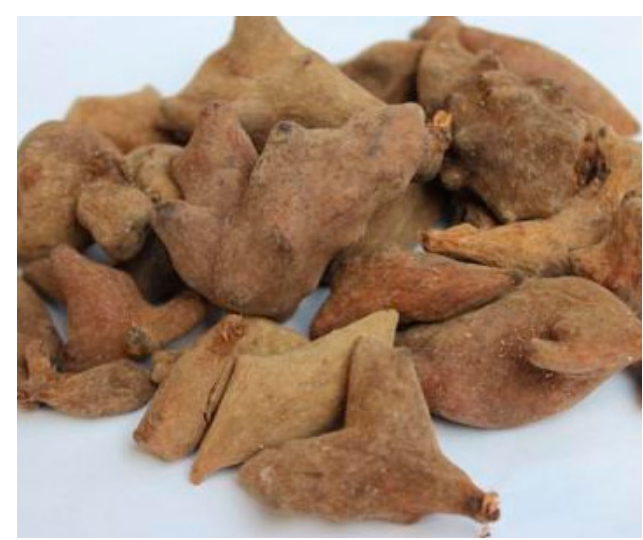

(b)

Figure 1. Chinese Du-ensiform gallnut, (a) fresh gallnut; (b) dry gallnut.

The enzymatic hydrolysis of GWE using the microorganisms of Aspergillus and Penicillium, particularly Aspergillus niger, has great potential for the industrialized production of gallic acid [3]. Wang [4] separated one strain of $A$. niger from Chinese Gall "Beihua" and fermented gallnut directly to produce GA with a yield of 78.5 89.5\%. Yang [5] used the same strain to ferment gallotannins and purified macroporous resin to obtain a high yield of $85.6 \%$ gallic acid. Banerjee modified a tannin-rich substrate of solid-state fermentation (MSSF) to produce tannase and gallic acid through co-culture method to obtain maximum yield of $94.8 \%$ gallic acid (GA) [6]. However, no literature has reported on fermented GWE chemical constitutes and structures so far, particularly with respect to the biodegradation mechanism of GWE biotransformation.

Since the microbial transformation of tannins was discovered by Tieghem [7], only a few analytical techniques, such as high-performance liquid chromatography (HPLC), thin-layer chromatography (TLC), or pyrolysis-gas chromatography-mass spectrometry (Py-GC/MS) have been applied to the identification of natural products and their metabolites by both conventional destructive or non-destructive methods in the process of chemical synthesis and biotransformation [8-11]. In order to develop gallic acid by microbial degradation of GWE, Min previously reported the extraction and screening of tannic acid degradation by the LHS-1 strain according to the indexes of tannic acid degradation rate, gallic acid accumulation concentration, and tannin enzyme activity [12]. This study focused on the optimization technology of LHS-1 strain degradation of GWE, and Py-GC/MS was applied to probe the chemical constituents and structural characterization of the linear and benzene ring derivatives, based on phenolic and glucose structure from fermented GWE (FGWE) pyrolysis. It will be very helpful for us to research the biodegradation mechanism and the corresponding metabolites of GWE for the future.

\section{Results}

\subsection{Determination of Tannic Acid and Gallic Acid in FGWE}

Folin-Ciocalteau and HPLC were both used to monitor the changes in gallic acid and tannic acid in GWE biodegradation with the LHS-1 strain. The standard curves of tannic acid and gallic acid had higher linearity. Tannic acid was detected at $630 \mathrm{~nm}$, and its equation was $y=69.848 x+0.0011$ $\left(R^{2}=0.9993\right)$, while the equation of gallic acid was $y=0.0029 x+0.0039\left(R^{2}=0.9998\right)$. The analysis showed GWE contained $81.6 \%$ of tannin acid and $5.6 \%$ of gallic acid. When GWE was incubated at $28{ }^{\circ} \mathrm{C}$ with a pH of 5.0 for $50 \mathrm{~h}$, HPLC showed that the levels of tannic acid decreased and those of gallic acid increased significantly in FGWE (Figure 2). 


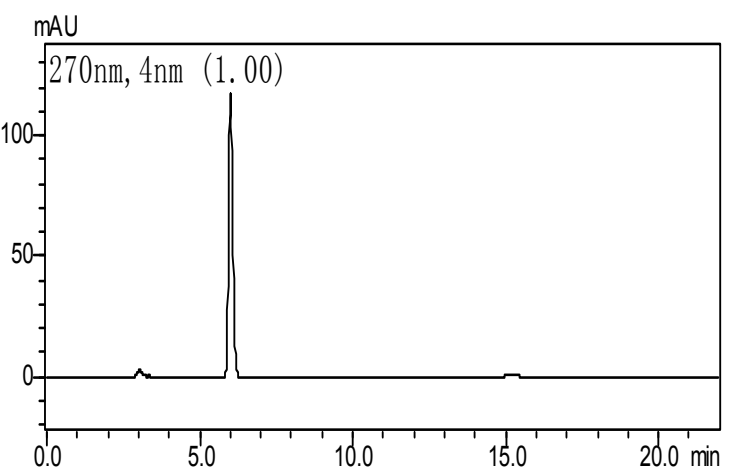

(a)

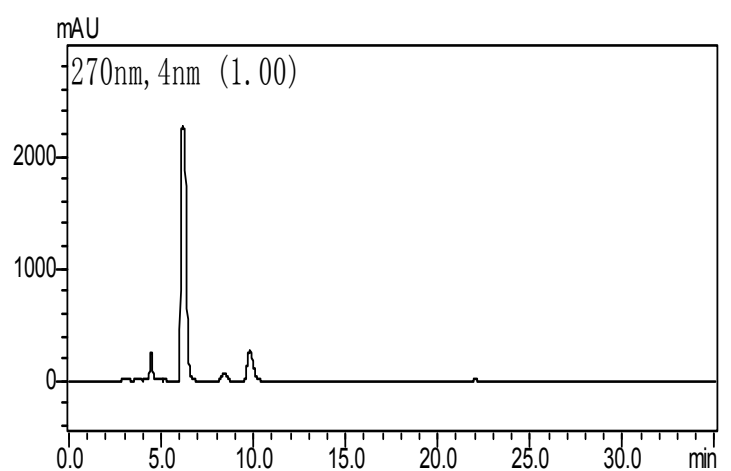

(b)

Figure 2. HPLC of a standard gallic acid sample and fermented gallnut water extract (FGWE), (a) standard sample of gallic acid; (b) the fermentation broth of GWE.

The degradation rate of GWE tannic acid exceeded $85.6 \%$. The concentration of gallic acid approached $63 \sim 65 \mathrm{mg} \cdot \mathrm{mL}^{-1}$, and tannin enzyme activity was $1.17 \mathrm{U} \cdot \mathrm{mL}^{-1}$. This indicated that the LHS-1 strain could produce tannase to transform tannic acid into gallic acid (Figure 3).

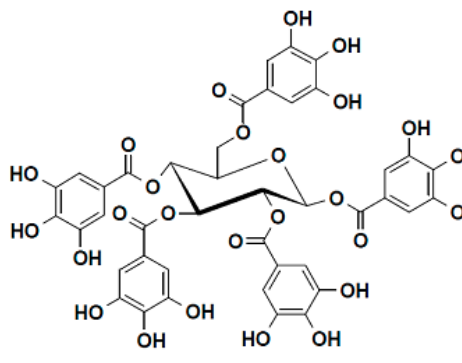

Tannic acid

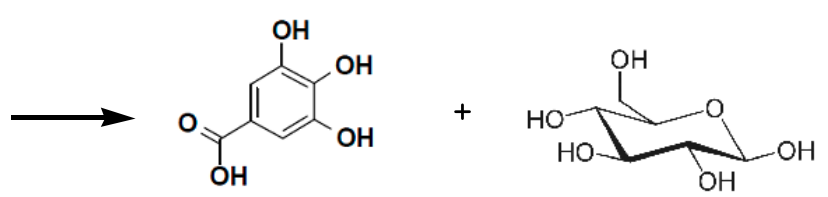

Gallic acid

Glucose

Figure 3. The degradation of GWE tannic acid into gallic acid.

\subsection{Box-Behnken Design and Variance Analysis of the LHS-1 Strain for Degrading Tannic Acid}

Since the incubation temperature, the initial $\mathrm{pH}$, and the incubation period obviously affected tannin enzyme activities in the culture media, and the ability of the LHS-1 strain to degrade tannic acid was measured through the degradation rate of tannic acid, the concentration of gallic acid, and the activity of tannase, the Box-Behnken experiment was designed and the results are shown in Table 1. The regression equation was determined as follows:

$$
\begin{aligned}
& Y=1.18+0.039 X_{1}+0.002 X_{2}+0.35 X_{3}-0.003 X_{1} X_{2}-0.007 X_{1} X_{3}+ \\
& 0.045 X_{2} X_{3}-0.10 X_{1}^{2}-0.13 X_{2}^{2}-0.15 X_{3}^{2}
\end{aligned}
$$

Variance analysis and significant tests of this regression model are shown in Table 2. The quadratic model was very significant and meaningful in statistics $(F=23.62, p=0.0002<0.001)$. The lack-of-fit item was good $(p=0.3680>0.05)$, and no loss factors existed. Therefore, the regression equation could substitute the real experiment to analyze and direct experiments. For the monomial items $\left(X_{1}, X_{2}\right.$, and $\left.X_{3}\right)$, the effects of culture temperature and incubation time on tannase activities were significantly different $(p<0.05)$. The effects of the three monomial factors on tannase activities ranging from strong to weak were in the following order: incubation temperature $\left(X_{1}\right)>$ incubation time $\left(X_{2}\right)>$ incubation initial $\mathrm{pH}\left(X_{3}\right)$. For interactive items of $X_{2} X_{3}(p=0.0352<0.05)$, initial incubation $\mathrm{pH}$ and the incubation time showed a great interaction, while $X_{1} X_{2}$ and $X_{1} X_{3}$ had no interaction $(p>0.05)$. For the binomial items of the equation, the effects of the incubation temperature, initial $\mathrm{pH}$, 
and incubation time on tannin enzyme activities reached significant and extreme levels $(p<0.0001)$. Through comparing the $p$-value, the primary and secondary factors could be judged for the LHS- 1 strain with respect to the degradation of tannic acid [13].

Table 1. The Box-Behnken experimental design and corresponding tannase activity for the LHS-1 strain.

\begin{tabular}{ccccc}
\hline No. & $\boldsymbol{X}_{\mathbf{1}}$ Temperature $\left({ }^{\circ} \mathbf{C}\right)$ & $\boldsymbol{X}_{\mathbf{2}}$ Initial $\mathbf{p H}$ & $\boldsymbol{X}_{\mathbf{3}}$ Incubation Time (h) & $\boldsymbol{Y}$ Tannase Activity $\left(\mathbf{U} \cdot \mathbf{m L}^{-\mathbf{1}}\right)$ \\
\hline 1 & -1 & -1 & 0 & 0.892 \\
2 & 1 & -1 & 0 & 0.968 \\
3 & -1 & 1 & 0 & 0.944 \\
4 & 1 & 1 & 0 & 1.016 \\
5 & -1 & 0 & -1 & 0.852 \\
6 & 1 & 0 & -1 & 0.948 \\
7 & -1 & 0 & 1 & 0.936 \\
8 & 1 & 0 & 1 & 1.004 \\
9 & 0 & -1 & -1 & 0.944 \\
10 & 0 & 1 & -1 & 0.812 \\
11 & 0 & -1 & 1 & 0.924 \\
12 & 0 & 1 & 1 & 0.972 \\
13 & 0 & 0 & 0 & 1.164 \\
14 & 0 & 0 & 0 & 1.14 \\
15 & 0 & 0 & 0 & 1.192 \\
16 & 0 & 0 & 0 & 1.22 \\
17 & 0 & 0 & 0 & 1.204 \\
\hline
\end{tabular}

Table 2. Variance analysis of items in the regression equation.

\begin{tabular}{cccccc}
\hline Sources of Variation & Sum of Squares & $d f$ & Mean Square & $\boldsymbol{F}$-Value & $\boldsymbol{p}$-Value \\
\hline model & 0.016 & 9 & 0.028 & 23.62 & 0.0002 \\
$X_{1}$ & $7.605 \times 10^{-5}$ & 1 & 0.012 & 10.19 & 0.0152 \\
$X_{2}$ & $2.000 \times 10^{-6}$ & 1 & $3.200 \times 10^{-5}$ & 0.027 & 0.8746 \\
$X_{3}$ & $6.125 \times 10^{-4}$ & 1 & $9.800 \times 10^{-3}$ & 8.21 & 0.0242 \\
$X_{1} X_{2}$ & $2.500 \times 10^{-7}$ & 1 & $4.00 \times 10^{-6}$ & 0.0033 & 0.9555 \\
$X_{1} X_{3}$ & $1.225 \times 10^{-5}$ & 1 & $1.960 \times 10^{-4}$ & 0.16 & 0.6975 \\
$X_{2} X_{3}$ & $5.063 \times 10^{-4}$ & 1 & $8.100 \times 10^{-3}$ & 6.78 & 0.0352 \\
$X_{1}^{2}$ & $2.819 \times 10^{-3}$ & 1 & 0.045 & 37.77 & 0.0005 \\
$X_{2}^{2}$ & $4.145 \times 10^{-3}$ & 1 & 0.066 & 55.53 & 0.0001 \\
$X_{3}^{2}$ & $5.571 \times 10^{-3}$ & 1 & 0.089 & 74.64 & $<0.0001$ \\
residual & $5.225 \times 10^{-4}$ & 7 & $1.194 \times 10^{-3}$ & & \\
lack-of-fit item & $2.665 \times 10^{-4}$ & 3 & $1.421 \times 10^{-3}$ & 1.39 & 0.3680 \\
pure error & $2.560 \times 10^{-4}$ & 4 & $1.024 \times 10^{-3}$ & & \\
total & 0.016 & 16 & & & \\
\hline
\end{tabular}

\subsection{The Optimization Technology for the LHS-1 Strain to Degrade Tannin}

According to the regression analysis and Box-Behnken experimental optimal model, the contour plots and 3D response surface plots were obtained by the Design Expert software, as shown in Figure 4, which are confined in the smallest ellipse in the contour diagram. The tannin enzyme activities were obtained with two continuous variables, while the other variables were fixed constantly. Elliptical contours were obtained when there was a perfect interaction between the independent variables. For $X_{2} X_{3}$ there existed a significant interaction $(p<0.05)$ and the contour map was shown to be elliptical. However, $X_{1} X_{2}$ and $X_{1} X_{3}$ showed round contours with no significant interaction $(p>0.05)$, indicating that $X_{1} X_{2}$ and $X_{1} X_{3}$ had less of an effect on tannin enzyme activities. Hence, initial $\mathrm{pH}$ and incubation time had more obvious effects. The maximum tannin enzyme activities were obtained when the temperature was around $29 \sim 33{ }^{\circ} \mathrm{C}$, with $\mathrm{pH}$ of $4.5 \sim 5.5$, and an incubation time of $42 \sim 54 \mathrm{~h}$. 
In the Box-Behnken design, the optimal conditions were determined as follows: cultivating temperature $30.92^{\circ} \mathrm{C}$, initial $\mathrm{pH} 5.03$, and incubation time of $49.44 \mathrm{~h}$, with the corresponding tannin enzyme activity of $1.192 \mathrm{U} \cdot \mathrm{mL}^{-1}$. Considering the practical experiments, the optimal parameters were adjusted to a temperature of $31^{\circ} \mathrm{C}$, initial $\mathrm{pH}$ of 5 , and incubation time of $50 \mathrm{~h}$, with tannin enzyme activity of $1.17 \mathrm{U} \cdot \mathrm{mL}^{-1}$. The relative error of the model was $1.68 \%(R<5 \%)$. Thus, the response model could reflect the expected optimization. Compared with an enzyme activity of $0.973 \mathrm{U} \cdot \mathrm{mL}^{-1}$, the LHS-1 strain could increase the tannin enzyme activity by more $20.2 \%$ in the optimal fermentation condition.

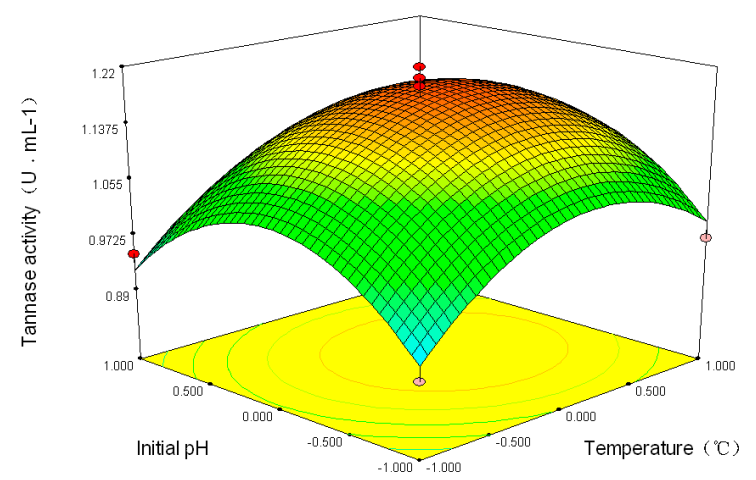

(a)

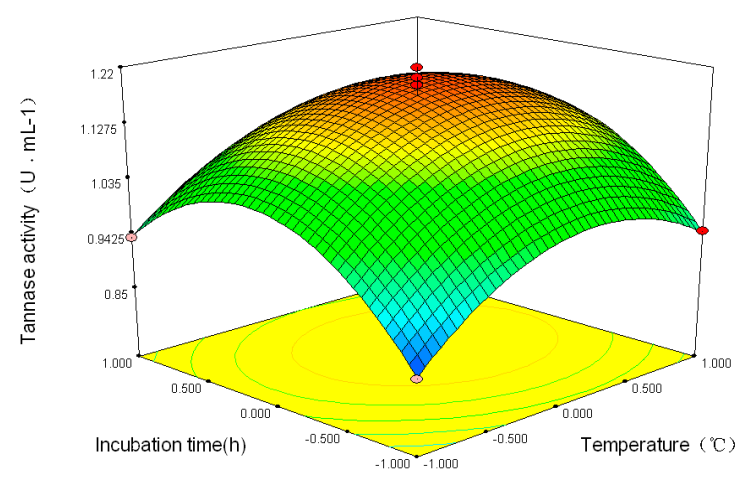

(b)

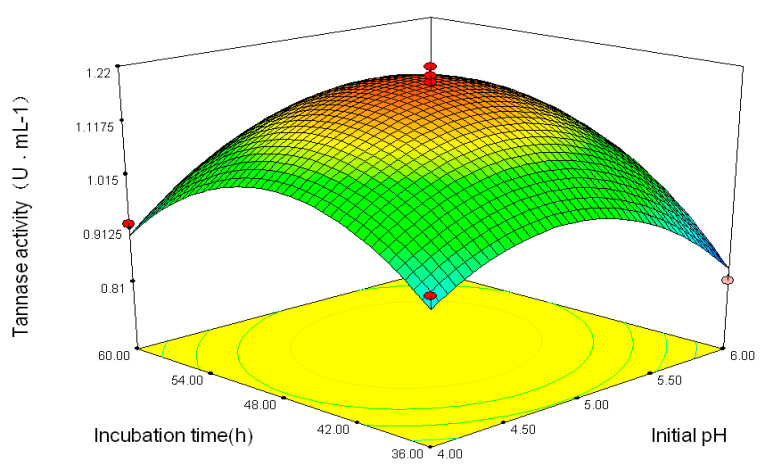

(c)
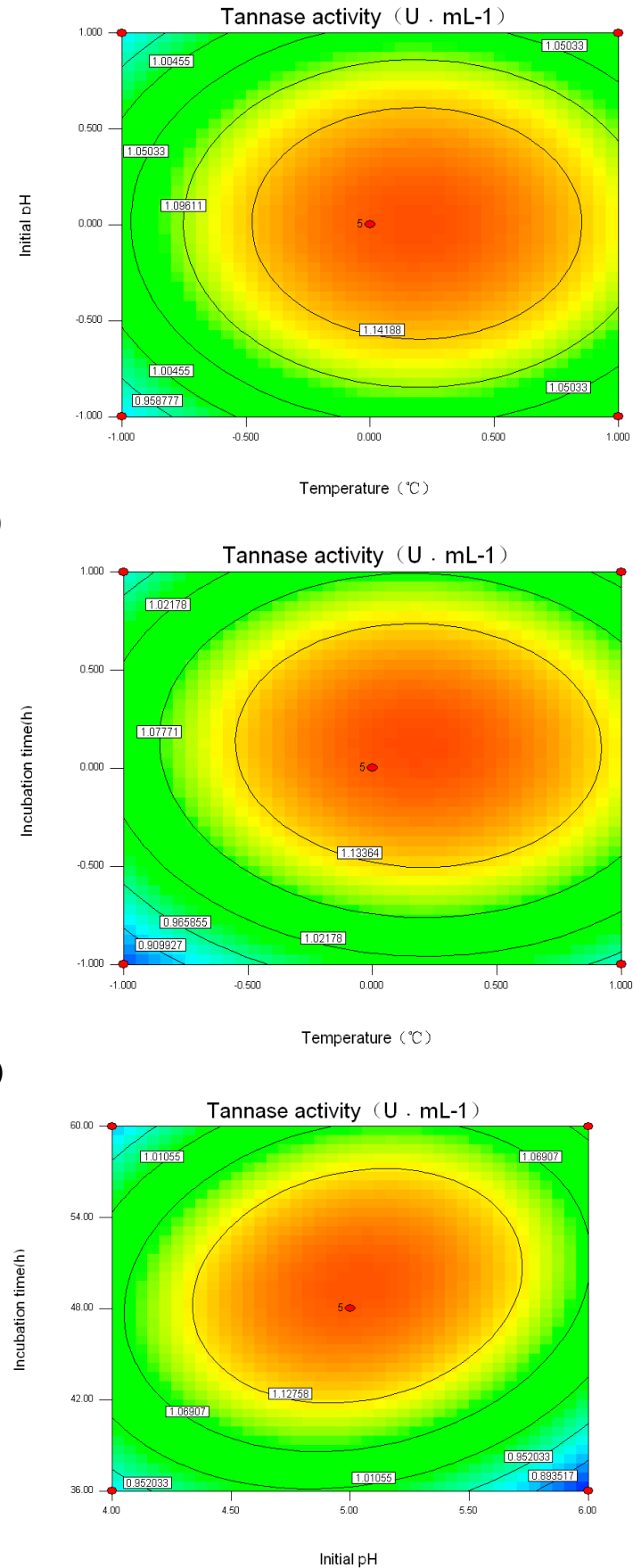

Figure 4. Contour plots and 3D-response surface plots showing (a) the interactive effects of incubation temperature and initial $\mathrm{pH}\left(X_{1} X_{2}\right)$; (b) incubation temperature and incubation time $\left(X_{1} X_{3}\right)$; and $(\mathrm{c})$ initial $\mathrm{pH}$ and incubation time $\left(X_{2} X_{3}\right)$. 
2.4. The Appraisal of Linear and Benzene Ring Derivatives Based on Phenolic and Glucose Structure from FGWE Pyrolysis by Py-GC/MS

Py-GC/MS was used to appraise the pyrolytic products of FGWE pyrolysis through comparison with the tannic acid standard sample (TAS) and GWE data, combined with GC-MS to determine gallic acid and tannic acid levels in samples. Their total ion chromatography peaks all consisted of linear and aromatic products, with retention times ranging from 9 to 13 min and 13 to 16 min, respectively, as well as gallic acid structural products with retention times ranging from 16 to $21.15 \mathrm{~min}$. Through comparison of the mass spectra of GC-MS retention times with corresponding standards, over 20 kinds of compounds were identified as linear and benzene ring matrix structures (Figure 5). The linear compounds, marked with numbers 1 9, were identified because of the pyrolysis of glucose, and benzene ring compounds were marked with numbers 10 20, which were appraised as the results of pyrolysis of ester bonds between gallic acid and glucose and epside bonds between gallic acids.

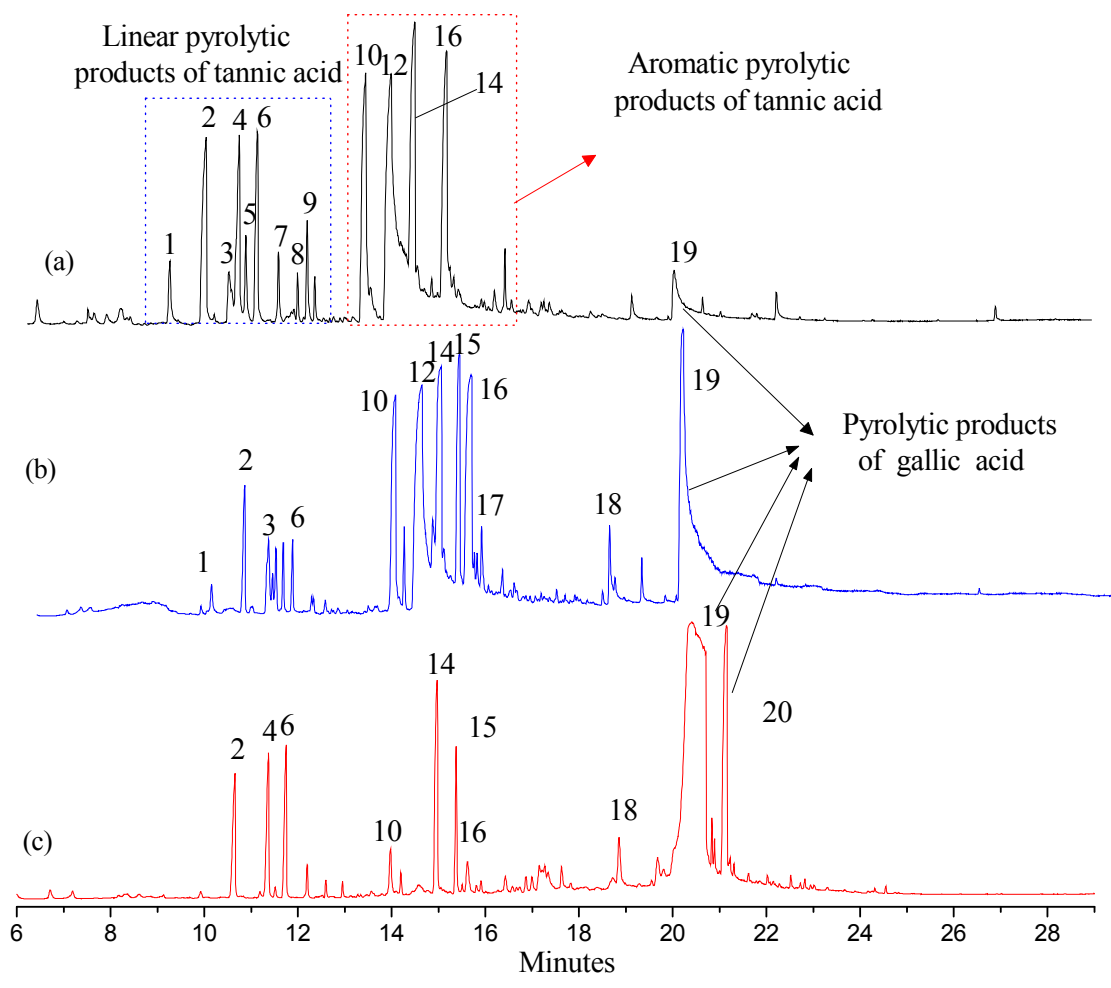

Figure 5. The total ion chromatography of three samples with pyrolysis-gas chromatography-mass spectrometry (Py-GC/MS). (a) Tannic acid standard sample (TAS); (b) GWE; (c) FGWE.

After pyrolysis, TAS produced 9 linear compounds and 5 benzene ring derivatives, while GWE pyrolyzed into 6 linear compounds and 10 aromatic ring products, and FGWE was found to produce only 3 linear compounds and 7 benzene ring derivatives. The differences originated from the contents of tannic acid and gallic acid. FGWE contained more gallic acid and little tannic acid, while TAS and GWE contained over $90 \%$ of tannin. Therefore, the results indicated that the LHS-1 strain could produce tannase to transform the tannic acid of GWE into gallic acid.

\subsection{The Pyrolytic Products of TAS, GWE, and FGWE}

The pyrolytic products of TAS, GWE, and FGWE were composed of linear and benzene ring compounds. The Py-GC/MS signal peaks integrated the related abundances of individual compounds and determined the pyrolytic products of TAS, GWE, and FGTA, as shown in Table 3. It can be seen that the pyrolytic products of TAS had 14 compounds, in 
which 3-methoxy-benzene-1,2-diol was $20.77 \%$ of the highest content, and the content of 2,6-dimethoxyphenol, 1,2,3-trimethoxybenzene and 3,4-dimethoxyphenol were between 11.18\% and $11.84 \%$. GWE contained analogous linear and benzene ring pyrolytic products such as TAS. Sixteen pyrolytic products were appraised in GWE pyrolysis. Among them, 3,4,5-trimethoxybenzoic acid methyl ester and 3-methoxy-1,2-benzenediol represented $13.77 \%$ and $12.70 \%$, respectively. Only four compounds represented between 5.32\% and 8.22\%. However, FGWE only produced pyrolytic products of 10 compounds. For FGWE, 3,4,5-trimethoxy-benzoic acid methyl ester represented $41.55 \%$ (the highest percentage), and 4-hydroxy-3,5-dimethoxy-benzoic acid hydrazide represented $7.42 \%$. The pyrolysis products of benzene ring derivatives from FGWE were further identified as 3,4-dimethoxy-phenol, 1,2,3-trimethoxybenzene, 3-methoxy-benzoic acid methyl ester, 2,6-dimethoxy-phenol, 3,4-dimethoxy-benzoic acid methyl ester, 3,4,5-trimethoxybenzoic acid methyl ester, and 4-hydroxy-3,5-dimethoxy-benzoic acid hydrazide. The contents of main pyrolytic products in TAS, GWE, and FGWE are shown in Figure 6.

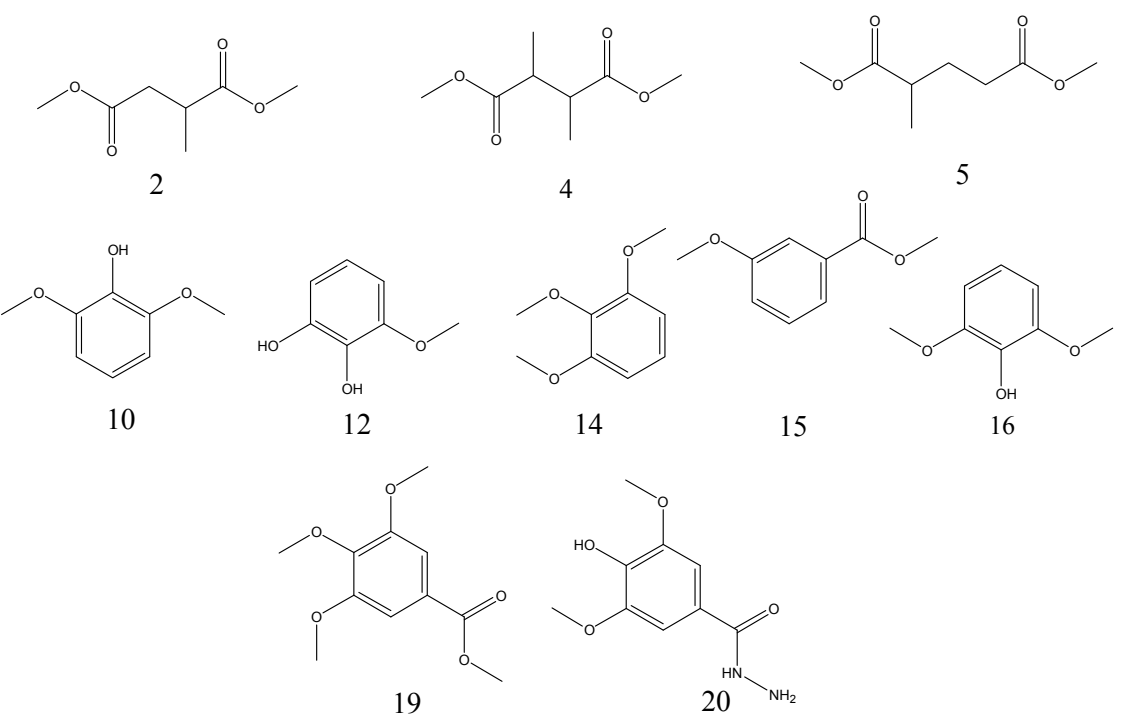

Figure 6. The chemical structures of the main pyrolytic products in TAS, GWE, and FGWE.

Table 3. The relative intensities of pyrolytic products of TAS, GWE, and FGWE.

\begin{tabular}{|c|c|c|c|c|c|c|c|}
\hline \multirow{2}{*}{ Peak } & \multirow{2}{*}{$\begin{array}{l}\text { Retention } \\
\text { Time (min) }\end{array}$} & \multirow{2}{*}{ MW } & \multirow{2}{*}{ Formula } & \multirow{2}{*}{ Compound } & \multicolumn{3}{|c|}{ Sample Total Peak Area, \% } \\
\hline & & & & & TAS & GWE & FGWE \\
\hline 1 & 9.946 & 146 & $\mathrm{C}_{6} \mathrm{H}_{10} \mathrm{O}_{4}$ & Succinic acid, dimethyl ester & 1.68 & 0.62 & - \\
\hline 2 & 10.656 & 160 & $\mathrm{C}_{7} \mathrm{H}_{12} \mathrm{O}_{4}$ & Succinic acid, methyl-, dimethyl ester & 8.11 & 1.8 & 2.57 \\
\hline 3 & 11.182 & 124 & $\mathrm{C}_{7} \mathrm{H}_{8} \mathrm{O}_{2}$ & Phenol, 2-methoxy- & 2.17 & 1.37 & - \\
\hline 4 & 11.348 & 174 & $\mathrm{C}_{8} \mathrm{H}_{14} \mathrm{O}_{4}$ & Dimethyl 2,3-dimethylsuccinate & 6.33 & 0.74 & 2.69 \\
\hline 5 & 11.520 & 158 & $\mathrm{C}_{7} \mathrm{H}_{10} \mathrm{O}_{4}$ & Dimethyl ethylidene malonate & 1.89 & 0.67 & - \\
\hline 6 & 11.749 & 174 & $\mathrm{C}_{8} \mathrm{H}_{14} \mathrm{O}_{4}$ & Pentane dioic acid, 2-methyl-, dimethyl ester & 5.35 & 0.71 & 2.62 \\
\hline 7 & 12.184 & 174 & $\mathrm{C}_{8} \mathrm{H}_{14} \mathrm{O}_{4}$ & Butane dioic acid, ethyl-, dimethyl ester & 1.30 & - & - \\
\hline 8 & 12.584 & 188 & $\mathrm{C}_{9} \mathrm{H}_{16} \mathrm{O}_{4}$ & Dimethyl 2-methyladipate & 0.73 & - & - \\
\hline 9 & 12.784 & 176 & $\mathrm{C}_{9} \mathrm{H}_{20} \mathrm{O}_{3}$ & 1,2,6-Trimethoxy-hexane & 1.86 & - & - \\
\hline 10 & 13.963 & 154 & $\mathrm{C}_{8} \mathrm{H}_{10} \mathrm{O}_{3}$ & Phenol, 3,4-dimethoxy- & 11.84 & 6.22 & 0.92 \\
\hline 11 & 14.009 & 152 & $\mathrm{C}_{9} \mathrm{H}_{12} \mathrm{O}_{2}$ & 3,5-Dimethoxytoluene & - & 0.81 & - \\
\hline 12 & 14.512 & 140 & $\mathrm{C}_{7} \mathrm{H}_{8} \mathrm{O}_{3}$ & 1,2-Benzenediol, 3-methoxy- & 20.77 & 12.70 & - \\
\hline 13 & 14.844 & 140 & $\mathrm{C}_{7} \mathrm{H}_{8} \mathrm{O}_{3}$ & 2-Methoxyresorcinol & - & 1.62 & - \\
\hline 14 & 14.964 & 168 & $\mathrm{C}_{9} \mathrm{H}_{12} \mathrm{O}_{3}$ & 1,2,3-Trimethoxybenzene & 11.18 & 7.63 & 4.39 \\
\hline 15 & 15.439 & 166 & $\mathrm{C}_{9} \mathrm{H}_{10} \mathrm{O}_{3}$ & 3-Methoxybenzoic acid methyl ester & - & 5.32 & 1.81 \\
\hline 16 & 15.640 & 154 & $\mathrm{C}_{8} \mathrm{H}_{10} \mathrm{O}_{3}$ & Phenol, 2,6-dimethoxy- & 11.24 & 8.22 & 0.71 \\
\hline 17 & 15.937 & 166 & $\mathrm{C}_{9} \mathrm{H}_{10} \mathrm{O}_{3}$ & Methyl 4-methoxybenzoate & - & 0.91 & - \\
\hline 18 & 18.793 & 196 & $\mathrm{C}_{10} \mathrm{H}_{12} \mathrm{O}_{4}$ & Benzoic acid, 3,4-dimethoxy-, methyl ester & - & 1.02 & 1.12 \\
\hline 19 & 20.383 & 226 & $\mathrm{C}_{11} \mathrm{H}_{14} \mathrm{O}_{5}$ & Benzoic acid, 3,4,5-trimethoxy-, methyl ester & 3.57 & 13.77 & 41.55 \\
\hline 20 & 21.150 & 212 & $\mathrm{C}_{9} \mathrm{H}_{12} \mathrm{~N}_{2} \mathrm{O}_{4}$ & Benzoic acid, 4-hydroxy-3,5-dimethoxy-, hydrazide & - & - & 7.42 \\
\hline
\end{tabular}




\section{Discussion}

Garnier et al. [8] reported temperatures effected on the pyrolytic process of hydrolysis-methylation of between $250{ }^{\circ} \mathrm{C}$ and $500{ }^{\circ} \mathrm{C}$. When the temperature was above $500{ }^{\circ} \mathrm{C}$, sufficient thermal energy probably led the formation of free radicals in the gas phase and caused thermal fragmentation reactions to occur. Therefore, we carried out pyrolytic processes with gallnut tannic acid at $380{ }^{\circ} \mathrm{C}$ for $5 \mathrm{~s}$ in order to control the radical fragmentations. All experiments were conducted in triplicate to study the reliability and stability, and the results provided enough evidence for good reproducibility of the method [10].

In general, Chinese gallnut contained 55 75\% tannic acid, and 2 5\% gallic acid and ellagic acid. GWE contained more than $90 \%$ of tannin [14]. Gallnut tannic acid is a $\beta$-glycoside bond polymer with one glucose core and five gallic acid esters. It is very easy for the LHS-1 strain or pyrolysis to break the ester bonds between the glucose core and gallic acid, like the benzoic acid and phenolic hydroxyl group. Those pyrolytic products with a benzene ring might be derived from splitting between the ester bonds in gallic acid and glucose, and the depside bonds among gallic acid, while liner pyrolytic products probably result because of the splitting among glucose.

Compared to TAS, GWE gave rise to linear and aromatic pyrolytic products of tannic acid as major products. It was obvious that both the varieties and quantities of linear products were reduced. Pyrolysis products like the ethyl-, dimethyl ester of butanedioic acid, dimethyl 2-methyladipate, and 1,2,6-trimethoxyhexane disappeared. While the aromatic products became more complex, new pyrolytic products, such as 3-methoxybenzoic acid methyl ester and 4-methoxy benzoic acid methyl ester, appeared. Benzoic acid 3,4,5-trimethoxybenzoic acid methyl ester had a greater presence, of about $13.77 \%$. All these results might be caused due to the effects of water extraction, which decreased the degrees of polymerization of tannic acid, resulting in an increase in free gallic acid and polyphenols so the structures of methylate with the benzene ring were more complex. Besides, the other water-soluble impurities mixed in the Chinese gall medicinal materials could influence the pyrolytic products.

However, the pyrolysis products of FGWE were further simplified as compared with TAS and GWE. The total abundances of 3,4,5-trimethoxybenzoic acid methyl ester and 4-hydroxy-3,5-dimethoxybenzoic acid hydrazide (pyrolysis and methylated products of gallic acid) were about $48.97 \%$, which was more than 3.6 times greater than in GWE. The results might be because there was no tannic acid with a high polymerization degree in FGWE. The LHS-1 strain degraded most of tannic acid in GWE into gallic acid and small amounts of low molecular weight phenolic substances and glucose in FGWE. Then, glucose continued to be degraded as the carbon source and formed the linear pyrolysis products. Meanwhile, FGWE contained few other sources of mycelium except the metabolite of the LHS-1 strain. These results showed that the LHS-1 strain gave a high degradation rate of tannic acid, resulting in the formation of gallic acid.

From the above, the biodegradation mechanism of gallnut tannic acid was different from that of the Chinese gallnut aqueous extract. Obviously, the differences in the pyrolytic products that existed in the three samples originated from the compositions of tannic acid and gallic acid. The benzene ring or aromatic compounds came from the pyrolysis of the epside bonds of gallic acid. The main pyrolytic products of FGWE were 3,4,5-trimethoxybenzoic acid methyl ester, 3-methoxy-1,2-benzenediol, and 4-hydroxy-3,5-dimethoxy- benzoic acid hydrazide.

\section{Materials and Methods}

\subsection{Raw Materials and Chemicals}

Chinese gallnut powder (containing $56.8 \%$ total tannic acid) was provided as a raw material by the Nanjing Longyuan Natural Polyphenol Synthesis Factory (Nanjing, China) in China, and was kept at $4{ }^{\circ} \mathrm{C}$ before used. The other chemicals were purchased from commercial suppliers (Sigma-Aldrich, St. Louis, MO, USA; Merck, Darmstadt, Germany; laddin, Beijing, China). GWE, FGWE, and the LHS-1 strain were prepared by our lab. 


\subsection{Basal Culture Mediums}

Plate sieve culture medium (tannic acid medium): The basal medium contained sucrose $20 \mathrm{~g}$, $\mathrm{NaNO}_{3} 2.0 \mathrm{~g}, \mathrm{~K}_{2} \mathrm{HPO}_{4} 1.0 \mathrm{~g}, \mathrm{MgSO}_{4} \cdot 7 \mathrm{H}_{2} \mathrm{O} 0.5 \mathrm{~g}, \mathrm{KCl} 0.5 \mathrm{~g}, \mathrm{FeSO}_{4} 0.01 \mathrm{~g}, 1 \%$ brom ophenol blue, gallnut tannin $10 \mathrm{~g}$, and agar $20 \mathrm{~g}$ in $1000 \mathrm{~mL}$ tap water.

Liquid fermentation medium: The basal medium contained sucrose $20 \mathrm{~g}, \mathrm{NaNO}_{3} 2.0 \mathrm{~g}, \mathrm{~K}_{2} \mathrm{HPO}_{4}$ $1.0 \mathrm{~g}, \mathrm{MgSO}_{4} \cdot 7 \mathrm{H}_{2} \mathrm{O} 0.5 \mathrm{~g}, \mathrm{KCl} 0.5 \mathrm{~g}, \mathrm{FeSO}_{4} 0.01 \mathrm{~g}$, and gallnut tannins $100 \mathrm{~g}$ in $1000 \mathrm{~mL}$ tap water. The above mediums were autoclaved at $121^{\circ} \mathrm{C}$ for $20 \mathrm{~min}$.

\subsection{Microorganism and Culture Conditions}

The LHS-1 strain was isolated from GWE in our laboratory previously, and was identified as a new variant of $A$. niger through traditional morphological identification and the phylogenetic tree of $18 \mathrm{~s}$ rDNA sequencing. It was then used for further study. The LHS-1 strain was kept for the culture of tannic acid agar slants stored at $4^{\circ} \mathrm{C}$ and sub-cultured for regular intervals of four weeks. The cells were grown at $30^{\circ} \mathrm{C}$ for 4 days shaking at $150 \mathrm{rpm}$.

\subsection{The Preparation of GWE and FGWE Samples}

Took $5 \mathrm{~g}$ of Chinese gallnut powder to mix with $50 \mathrm{~mL}$ distilled water in a $150-\mathrm{mL}$ extractor at $50{ }^{\circ} \mathrm{C}$ for $1 \mathrm{~h}$ by vacuum cavitation extraction, then filtered and centrifuged. The filtrate was freeze-dried at $-50{ }^{\circ} \mathrm{C}$ for $72 \mathrm{~h}$ to obtain GWE, and stored at $4{ }^{\circ} \mathrm{C}$ before use.

$250 \mathrm{~mL}$ of GWE solution was then sterilized at $121^{\circ} \mathrm{C}$ for $20 \mathrm{~min}$. After being cooled to room temperature, the inoculated strain LHS-1 was added to the sterilized GWE solution, and the mixture was shaken at $150 \mathrm{rpm}$ at $30^{\circ} \mathrm{C}$ for 4 days. After the GWE was fermented by the LHS- 1 strain, the fermented broth was filtered in a vacuum, and the filtrate was freeze-dried at $-50{ }^{\circ} \mathrm{C}$ until the LHS-1 strain fermentation sample of GWE (FGWE) was obtained. The sample was then stored at $4{ }^{\circ} \mathrm{C}$.

\subsection{Analysis of Tannic Acid, Gallic Acid, and Tannin Enzyme Activities}

Tannic acid was analyzed using Folin-Ciocalteau on the basis of the standard curve generated with tannic acid standard [9-11]. HPLC was used to assay tannic acid and gallic acid. The HPLC conditions were as follows: C18 $(150 \mathrm{~mm} \times \Phi 4.6 \mathrm{~mm} \times 5 \mu \mathrm{m})$ chromatographic column, PDA detector, methanol-water $(0.5 \%)=5: 95$ of mobile phase, $270-\mathrm{nm}$ wavelength determination, $1 \mathrm{~mL} \mathrm{~min}^{-1}$ of flow speed.

The degradation rate of tannic acid was calculated with the formula: $R=1-\frac{T A_{t}}{T A_{0}}$, where $R$ is degradation rate of tannic acid, $T A_{0}$ is the concentration of tannic acid in the TAA medium, and $T A_{t}$ is the concentration of tannic acid at the time of $\mathrm{T}(\mathrm{t})$.

A small amount of nutrient solution was taken, and the mycelium filtered as a crude enzyme liquid. Three tubes were marked as the blank, test, and control tubes, respectively. Gallic acid accumulation and tannase activities were assayed by the spectrophotometric method of methanolic rhodanine [15]. One unit (U) of tannase was defined as one micromole of gallic acid formed per minute. Tannin enzyme activities were determined according to the content of TA and GA.

\subsection{The Box-Behnken Experiment on GWE Biodegradation}

For determining the optimum parameters of GWE biodegradation with the LHS-1 strain, a Box-Behnken experiment was designed with three factors and three levels (Table 4). The parameters were studied with ranges as follows: temperature of $25 \sim 35^{\circ} \mathrm{C}$, initial $\mathrm{pH}$ of $4 \sim 6$, and incubation period of $36 \sim 60 \mathrm{~h}$. 
Table 4. Factors and levels in response surface analysis.

\begin{tabular}{ccccc}
\hline \multirow{2}{*}{ Factors } & Code & \multicolumn{3}{c}{ Levels } \\
\cline { 3 - 5 } & & $\mathbf{- 1}$ & $\mathbf{0}$ & $\mathbf{1}$ \\
\hline A: temperature $\left({ }^{\circ} \mathrm{C}\right)$ & $X_{1}$ & 25 & 30 & 35 \\
B: initial $\mathrm{pH}$ & $X_{2}$ & 4 & 5 & 6 \\
C: incubation period $(\mathrm{h})$ & $X_{3}$ & 36 & 48 & 60 \\
\hline
\end{tabular}

\subsection{Conditions of the Py-GC/MS Experiments}

Py-GC/MS was performed using a double-shot pyrolyzer (Frontier Laboratories, model 2020i) attached to a GC/MS system Agilent 6890N (Santa Clara, CA, USA). TAS, GWE, and FGWE samples were analyzed by Py-GC/MS in accordance with procedures.

Samples of $0.4 \mathrm{mg}$ of TAS, GWE, and FGWE were placed in their respective sample cups. Sufficient methylated derivatives were added with a microsyringe (TMAH, 25\% methanol), and then the samples were placed in small crucible capsules and introduced into the furnace, which was preheated at $500{ }^{\circ} \mathrm{C}$ for $1 \mathrm{~min}$. GC-MS analysis with the Agilent $6890 \mathrm{~N}$ system was performed. The GC was equipped with a low-to-mid polarity-fused silica capillary column of HP-5 of $30 \mathrm{~m} \times 250 \mu \mathrm{m} \times 25 \mu \mathrm{m}$ film thickness. Column temperature: initial temperature $50{ }^{\circ} \mathrm{C}$ for $5 \mathrm{~min}$, with a subsequent increase rate of $10^{\circ} \mathrm{C} / \mathrm{min}$ until $280^{\circ} \mathrm{C}$, maintained for $15 \mathrm{~min}$. The vaporizing chamber temperature was $300{ }^{\circ} \mathrm{C}$ and the carrier gas was helium. There was a constant pressure mode $(6.0 \mathrm{kPa})$, and a split ratio of 20:1. Pyrolysis temperature was $380^{\circ} \mathrm{C}$ for $5 \mathrm{~s}$. The detector consisted of an Agilent 5975 mass selective detector and the electrical energy acquired was $70 \mathrm{eV}$. The compounds were identified by comparing their mass spectra with reference compounds from the $\mathrm{NISTO}_{2}$ and Wiley libraries. Traces corresponding to selected homologous series of chemical families were obtained by single ion monitoring (SIM) of characteristic ions.

\section{Conclusions}

TAS, GWE, and FGWE samples were analyzed using HPLC and Py-GC/MS. The optimization technology of the LHS-1 strain degrading GWE was determined by a response surface method. The optimum fermentation conditions of the LHS-1 strain were as follows: temperature of $31^{\circ} \mathrm{C}$, $\mathrm{pH}$ of 5 , and a $50-\mathrm{h}$ fermentation time. The tannase activity was $1.17 \mathrm{U} \cdot \mathrm{mL}^{-1}$. Through comparing the mass spectra of analytes in NITS02 libraries and the retention times with the corresponding standard references, over 20 kinds of compounds were determined and annotated on the chromatogram. The pyrolytic products of all three samples were shown to be composed of linear and aromatic compounds. Among these, the total abundance of benzene ring derivatives of FGWE was about $48.97 \%$. The metabolites of the LHS-1 strain contained almost no high polymerized tannic acid, giving a high degradation rate of tannic acid resulting in the formation of gallic acid and small amounts of phenolic compounds and glucose. Py-GC/MS is a convenient and efficient method for tracing tannic acid and gallic acid in fermentation broth, and has significant applicable value.

Acknowledgments: This work was supported by the work was supported by the National Key Research \& Development Program (No. 2016YFD0600805) and National Natural Science Foundation of China (No. 31570564).

Author Contributions: Chengzhang Wang and Wenjun Li conceived and designed the experiments; Wenjun Li performed the experiments; Wenjun Li analyzed the data; Chengzhang Wang contributed reagents/materials/analysis tools; Chengzhang Wang and Wenjun Li wrote the paper.

Conflicts of Interest: The authors declare no conflict of interest. 


\section{References}

1. Aguilar, C.N.; Rodríguez, R.; Gutiérrez-Sánchez, G.; Augur, C.; Favela-Torres, E.; Prado-Barragan, L.A.; Ramírez-Coronel, A.; Contreras-Esquivel, J.C. Microbial tannase: Advances and perspectives. Appl. Microbiol. Biotechnol. 2007, 76, 47-59. [CrossRef] [PubMed]

2. Chávez-González, M.; Rodríguez-Durán, L.V.; Balagurusamy, N.; Prado-Barragán, A.; Rodríguez, R.; Contreras, J.C.; Aguilar, C.N. Biotechnological advances and challenges of tannase: An overview. Food Bioprocess Technol. 2012, 5, 445-459. [CrossRef]

3. Van Diepeningen, A.D.; Debets, A.J.M.; Varga, J. Efficient degradation of tannic acid by black Aspergillus species. Mycol. Res. 2004, 108, 919-925. [CrossRef] [PubMed]

4. Wang, W.W. Preparation of gallic acid by high conversion rate biological method. For. Chem. Commun. 1996, 1, 3-6.

5. Yang, X.K. Enzymatic Synthesis of Gallic Acid from Tannic Acid. Fine Spec. Chem. 2005, 5, 12-15.

6. Rintu, B.; Gargi, M.; Krushan, C.P. Microbial transformation of tannin-rich substrate to gallic acid through co-culture method. Bioresour. Technol. 2005, 96, 949-953.

7. Tieghem, P. Sur la Fermentation Gallique. Comptes Rendus Hebdomadaires des Seances de I'Academie des Sciences. 1867, Volume 65, pp. 1091-1094. Available online: http://gallica.bnf.fr/ark: /12148/bpt6k3021f.image.r=Comptes++Rendus+Hebdomadaires+des+S\%C3\%A9ances+de+1\%E2\%80\% 99Acad\%C3\%A9mie+des+Sciences.f837.langFR (accessed on 16 December 2017).

8. Garnier, N.; Richardin, P.; Cheynier, V.; Regert, M. Characterization of thermally assisted hydrolysis and methylation products of polyphenols from modern and archaeological vine derivatives using gas chromatography-mass spectrometry. Anal. Chim. Acta 2003, 493, 137-157. [CrossRef]

9. Kuroda, K.; Nakagawa-izumi, A.; Mazumder, B.; Ohtani, Y.; Sameshima, K. Evaluation of chemical composition of the core and bast lignins of variety Chinpi-3 kenaf (Hibiscus cannabinus L.) by pyrolysis-gas chromatography/mass spectrometry and cupric oxide oxidation. Ind. Crops Prod. 2005, 22, 223-232. [CrossRef]

10. Wang, L.L.; Qian, J.; Hu, Z.C.; Zheng, Y.G.; Hu, W. Determination of dihydroxyacetone and glycerol in fermentation broth by pyrolytic methylation/gas chromatography. Anal. Chim. Acta 2006, 557, 262-266.

11. Cai, X.H.; Liu, Y.R.; Tian, S.B.; Zhou, J.; Liu, Z.L. Direct fingerprinting polycyclic aromatic hydrocarbons in heavy oils by PY-GC/MS and its application to hydrotreating processes. China Pet. Process. Petrochem. Technol. 2012, 14, 8-14.

12. Min, F.Q. Screening of Tannic Acid Degradation Strains and Metabolites Research. Master's Thesis, Chinese Academy Forestry, Beijing, China, 2014.

13. Zhang, L.L.; Xu, M.; Wang, Y.M.; Wu, D.M.; Chen, J.H. Optimization of Ultrasonic Extraction Conditions of Ellagic Acid from Infructescence of Platycarya strobilacea Using Response Surface Method. Chem. Ind. For. Prod. 2011, 31, 19-24.

14. Liu, S.P.; Li, Y. Study on the Extraction of Tannic acid from Chinese Gallnut. He Bei Chem. Eng. Ind. 2007, 20, $44-45$.

15. Bao, Y.X.; Qiu, S.Y.; Li, Y.Z.; Huang, Y.G. A Method for Detecting Activity of Extracellular Tannase. Fine Chem. 2008, 25, 621-624.

Sample Availability: Samples of the compounds are available from the authors. 